\title{
Study on Nearby Residents and Quantify the Waste Incineration Factory
}

\author{
Xie Zhou ${ }^{1, *}$, Xingneng Xia ${ }^{2}$ \\ ${ }^{1}$ Economics College, Shanghai University, Shanghai, China \\ ${ }^{2}$ Management College, Xi'an University of Science and Technology, Xi'an, China
}

\section{Email address:}

1746553175@qq.com (Xie Zhou),876314690@qq.com (Xingneng Xia)

*Corresponding author

\section{To cite this article:}

Xie Zhou, Xingneng Xia. Study on Nearby Residents and Quantify the Waste Incineration Factory. Science Innovation. Vol. 5, No. 3, 2017, pp. 132-137. doi: 10.11648/j.si.20170503.12

Received: March 31, 2017; Accepted: April 17, 2017; Published: April 20, 2017

\begin{abstract}
With the extension of the urban fringe and the expansion of the scale of the city, the daily output of domestic waste is also increasing. While the city people want to raise the quality of life requirements, it is hoped that the government can build a landfill, but do not want to refuse to live next to the landfill. Residents and the government can not accurately understand the residents in the vicinity of the incineration plant suffered losses. Once you can quantify the loss of residents, the government knows its subsidies to deal with, residents clearly know their income. This allows them to make their own most favorable decisions to improve their situation.
\end{abstract}

Keywords: Incineration Plant, Resident, Game Theory, Cost, Benefit

\section{量化垃圾焚烧厂对附近居民损益的研究}

周䃞 ${ }^{1 *}$, 夏兴能 ${ }^{2}$

'经济学院, 上海大学, 上海, 中国

2管理学院, 西安科技大学, 西安, 中国

邮箱

1746553175@qq. com（周誉）,876314690@qq. com(夏兴能)

摘要: 随着城市边缘的外延和城市规模的扩大, 城市中每日生活垃圾的产出量也日渐增加。而城市人们要求生活质量 要求的上升, 人们希望政府能修建垃圾填埋场, 又不希望垃圾与垃圾填埋场比邻而居。居民和政府都无法准确的了解 居民在焚烧厂附近所受到的损失。一旦可以量化出居民的损失，政府了解出其应付的补贴，居民清晰知道自己收益。 这样能够让他们做出对自己最有利的决策来改善自己的情况。

关键词: 焚烧厂, 居民, 博弯, 成本, 收益

\section{1. 引言}

当今中国, 政府不断强调城镇化, 城市的规模变得越 来越大，同时也给城市带来了垃圾围城的问题。人们希望
政府能快速的处理掉垃圾来改善大家的生活环境, 但是又 不愿意自己成为那个垃圾处理危害的受害者。纵观近年来 发生的典型邻避事件, 几乎每一起都是由利益失衡、沟通 不畅、信任缺失、情绪焦虑等多重因素叠加所致, 公众焦 
虑程度与项目的接受度相关。焦虑程度越高的公众, 越不 倾向于支持在周边建设项目。焦虑情绪持续放大, 积累到 一定程度, 就会在一瞬间爆发出来, 出现后果难测的冲动 行为。[1]在多重的要求下, 焚烧厂变成了居民和政府矛 盾的关键所在。本文从居民的经济、健康和心理三个角度 去量化他们的损益情况。运用特征价格法去分析焚烧厂对 附近小区房价的影响来量化居民所受经济损失。结合相关 的法律、法规和现有医疗情况来评估焚烧厂对附近居民健 康的影响。用马斯洛需求层次理论评估居民心理。从量化 的结果能够更加直观的看到居民受到焚烧厂的危害, 同时 让政府也能准确的了解焚烧厂对附近居民的影响。可以帮 助政府和居民找到合理解决邻避效应的方案, 最终能够让 居民能够接受焚烧厂。

\section{2. 附近居民的经济损益函数}

\section{1. 研究方法}

研究住宅价格影响的最重要方法是特征价格模型。在 Lancaster的消费者理论中, 商品具有一系列特性主要特 征, 而这些特征通过不同的组合来影响消费者的选择, 因 此, 由于住宅价格的不同特征的组合而变得不同。在对焚 烧厂邻避效应的研究中, 国内学者在这方面的研究刚刚起 步, 并且定性分析、定量研究明显不足。第一部分就是考 虑了住宅市场中垃圾焚烧厂的周边环境、区位选择、建筑 物结构和邻里环境三种特征变量的住宅价格、特征定价模 型和实证研究。[2]

（1）特征价格法的理论基础

特征价格法的基本思路是: 将房地产商品的价格分解, 以显现出其各项特征的隐含价格, 在保持房地产的特征不 变的情况下, 将房地产价格变动中的特征因素分解, 从价 格的总变动中逐项剔除特征变动的影响, 剩下的便是纯粹 由供求关系引起的价格变动。

特征价格法: 该理论认为, 一种多样性商品具有多方 面的不同特征或品质 (如房地产商品的面积、楼层、朝向 和是否有保安服务等特征), 商品价格则是所有这些特征 的综合反映和表现。当商品某一方面的特征改变时, 商品 的价格也会随之改变。

当商品的某一方面的特征改变时, 其价格也会随之发 生改变。对函数的各个特征变量分别求偏导数, 就取得各 特征的变动对商品价格的影响幅度, 并假定这种影响的关 系在一定的时间内固定不变。这样, 在缺乏同质商品的情 况下, 可以用非同质的房地产在基期与报告期之间进行比 较, 从价格的总变动中逐项剔除特征变动的影响, 最后剩 下的便是纯粹由供求关系引起的价格变动了, 这样计算的 价格指数便是基于特征价格法的房地产价格指数。

(2) 特征价格法的主要形式

a. 时间亚元法

其基本思路是, 利用基期和多个报告期的样本建立统 一的特征价格模型, 并在模型中以时间亚元变量标识样本 所属的报告期。

b. 特征价格指数法
其基本思路是, 各个报告期分别建立特征价格模型, 以计算各住房特征在各报告期内的特征价格值, 再选定一 定的 “标准单元”（即固定各住房特征在各报告期内的取 值）。将计算得到的特征价格值和设定的标准单元值分别 代入（2）后，即可得到 “标准单元” 在各报告期的同质 价格, 并以此为基础进行指数编制。

\section{c. 模拟价格法}

模拟价格法是基于特征价格模型对样本匹配法的改 进。样本匹配法是普通商品价格统计中的一种常用方法, 其基本思路是严格控制考察样本在各报告期内一致, 以满 足同质可比的要求。但由于住房具有高度异质性特点，同 一住房单元几乎不可能在各报告期内均发生交易, 而各期 交易的住房单元在质量上又存在不可忽略的差异, 因此传 统的样本匹配法不能简单套用到住房价格指数编制过程 中。

模拟价格法对样本匹配法的改进思路是，计算第 $\mathrm{t}$ 期 指数时, 首先利用第 $t-1$ 期的特征价格模型, 推测第 $\mathrm{t}$ 期 交易的住房单元（相当于第 $t$ 期的 “新增单元” ) 在第 $t-1$ 期的价格, 再利用第 $\mathrm{t}$ 期的特征价格模型, 推测第 $\mathrm{t}-1$ 期交 易的住房单元（相当于第 $t$ 期的 “退出单元” ) 在第 $t$ 期的 价格, 从而实现第 $t-1$ 期和第 $t$ 期内住房单元的完全匹配, 进而引入传统的样本匹配法进行指数计算。

\section{d. 价格调整法}

价格调整法同样是利用特征价格模型对样本匹配法 进行的一种改进。其基本思路是, 确定某一虚拟的 “标准 住房单元” 作为匹配样本, 在各报告期内, 均利用当期的 特征价格模型, 将所有交易的住房单元的价格调整到统一 的 “标准住房单元” 的基础上, 再利用样本匹配法进行指 数编制。

（3）特征价格法具体形式的选择

由于基本思路的差异，不同形式的特征价格法各有其 利弊和适用范围, 这也使得具体形式的选择成为特征价格 法应用中的首要问题。

首先是直接特征价格法和间接特征价格法的选择。在 住房价格指数编制中, 间接特征价格法需要对各住房单元 逐一进行模拟或调整, 因此相比之下间接特征价格法的计 算量明显超过直接特征价格法, 但结果的准确程度反而可 能更低, 从效果上看并非理想选择。因此, 直接特征价格 法目前占据了主流地位, 而间接特征价格法的应用则主要 限于对正在使用的非同质方法进行改进，例如德国统计部 门正在进行的基于价格模拟法的住房价格指数编制研究 等。

就直接特征价格法而言, 时间亚元法和特征价格指数 法也各有其显著特点。时间哑元法在特征价格法的各种形 式中计算最为简便, 且通过多期样本的叠加, 能够有效的 避免样本代表性误差, 其指数结果具有较好的稳健性。但 如前所述, 时间亚元法的前提假设是各住房特征的特征价 格在各报告期内保持不变, 当考察期延长, 或当市场处于 剧烈变化过程中时, 这一假设很有可能无法成立, 并将因 此造成误差; 其次, 时间亚元法在进行指数更新时存在一 定的困难, 引入新的报告期时, 估计得到的特征价格模型 将发生改变, 并使历史指数值也发生变化。特征价格指数 法则可以有效的改善时间亚元法的这两方面不足, 但该方 
法的稳健性较差, 各报告期中的特征价格估计值容易由于 交易结构波动、样本容量不足或数据质量的微小误差而出 现较大幅度的变化, 并因而造成指数序列的震荡。由此可 见，这两种方法不存在绝对的优劣之分，而适用于不同的 市场情况。

另一种可行的思路是将时间亚元法和特征价格指数 法相结合, 取长补短。目前较为常见的是链式指数法, 这 种方法仍以式 (1) 为基本形式, 但在计算第 $\mathrm{t}$ 期价格指数 时, 仅引入第 $\mathrm{t}$ 期和第 $\mathrm{t}-1$ 期的样本, 再通过时间哑元变量 系数计算第 $\mathrm{t}$ 期价格指数相对于第 $\mathrm{t}-1$ 期的变化程度 (即环 比值），最后利用第 $\mathrm{t}-1$ 期价格指数和该环比值推算第 $\mathrm{t}$ 期价格指数。链式指数法同样有效的避免了时间哑元法存 在的两方面缺陷, 同时与特征价格指数法相比, 它通过两 期样本的叠加, 在一定程度上提高了结果的稳健性。在此 基础上, 对其进行了进一步发展, 提出了扩展的链式指数 法, 在结果的稳健性程度等方面又有所提高。

\section{2. 研究区域与数据采集}

(1) 研究区域概况

上海地区有四间焚烧厂，它们分别是江桥焚烧厂、御 桥焚烧厂、曹杨焚烧厂、老港焚烧厂。选取的是这江桥焚 烧厂三公里范围内的小区综合讨论, 讨论出焚烧厂对居民 的经济影响。

(2) 数据的采集与处理

本文采集了江桥焚烧厂三个住宅板块 24 个住宅小区 共442套住宅案例用作实证研究, 选取2016年1月至2016 年11月主要房产板块的每月平均房价进行分析, 具体样本 数据见表2。所选取的住宅案例均是普通的商品住房, 包
括多层、小高层以及高层住宅，但不包括复式结构、跃层 结构等形式的住宅, 别墅、经济适用房等也均不予考虑。 研究样本数据的来源主要有: a. 二手房交易价格从上海 58 安居客shanghai. an juke. com和其他在嘉定房地产中介机 构; b. 从《上海楼市》海房地产市场获取商品房的价格; c. 用百度测量的距离测量焚烧厂到住宅区的距离; d. 住宅 周围的环境、配套设施（学校、商务中心等）、交通（地 铁、公交）、装修施工、物业管理费，从上海的58安居客 shanghai. anjuke. com获取, 同时还进行必要的实地调查。

\section{3. 上海焚烧站周边住宅价格的空间效应分析}

特征价格模型的建立需要选择必要的特征变量。住宅 的特征一般分为三种类型: a. 区位特征, 即量化的可达性, 包括交通便利、出行时间、出行成本等特点; b. 住宅建筑 的自身特点, 有些特点包括定位、朝向、装饰、房龄、车 位; c. 特色街区, 涉及住宅周围的生活环境是购房者密切 相关的特性, 包括空气质量、社区绿化率、生活配套设施、 物业管理费等。[2]

\section{（1）特征变量的选择与定量}

本文选取涉及上述三大类的特征变量共 11 个, 包括住 宅房龄、装修程度、卧室个数、卫生间个数、朝向、是否 具有停车位、交通条件、到自贸区中心的距离、小区绿化 率、物业管理费与生活配套, 并对其进行必要的定量描述。

(2) 数据分析

a. 数据的预处理

本文总共收集了 442 套房产数据, 因交易日期各不相 同, 以中房上海住宅指数对住宅价格进行修正, 将其统一 归化到2016年12月。

表1 住宅小区特征变量及其定量描述。

\begin{tabular}{|c|c|c|c|}
\hline 特征变量 & & 内涵 & 定量描述 \\
\hline 建筑特征 & $\begin{array}{l}\text { 房龄 } \\
\text { 装修程度 } \\
\text { 卧室个数 } \\
\text { 卫生间个数 } \\
\text { 朝向 } \\
\text { 停车位 }\end{array}$ & \multirow{3}{*}{$\begin{array}{l}\text { 住宅的军工年份, 单位年 } \\
\text { 住宅的装修情况 } \\
\text { 住宅的厅室情况 } \\
\text { 住宅的卫生间情况 } \\
\text { 住宅的采光情况 } \\
\text { 住宅是否配套停车位 } \\
\text { 从住宅小区步行到最近的地铁 } \\
\text { 或是公交站所花的出行时间 } \\
\text { 住宅小区到焚烧厂的距离 } \\
\text { 住宅小区的绿化情况 } \\
\text { 反映住宅小区的物业管理水平 }\end{array}$} & $\begin{array}{l}\text { 进行折旧修正 } \\
\text { 分为四级: 毛坯房 (0分), 简装修 (1分), 中装修 (2分), 精装修 (3分) }\end{array}$ \\
\hline 区位特征 & 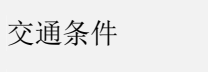 & & 分为三级: 10 分钟以内 (3分), 10 到 20 分钟 (2分), 30 分钟以上 (0分) \\
\hline & $\begin{array}{l}\text { 到焚烧厂的距离 } \\
\text { 小区绿化率 } \\
\text { 物业管理费 }\end{array}$ & & 以 $\mathrm{km}$ 为单位 \\
\hline 邻里特沚 & 生活配套 & 住宅小区周边的生活环境 & $\begin{array}{l}\text { 该项按照以下方式评分, 住宅小区周边是否有学校、医院、商场, 每有一 } \\
\text { 项, 增加 } 1 \text { 分, 满分 } 3 \text { 分, 最低分 } 0 \text { 分 }\end{array}$ \\
\hline
\end{tabular}

经比较分析, 认为采用半对数形式的特征价格模型比 较适合, 并设模型为: 其中: $C$ 为常数, $\mathrm{P}$ 为修正后的住房 价格, 以对数表示; B1为房龄, 以年为单位; B2为生活配 套; B3为交通条件; B4为物业管理费, 单位为元 $/ \mathrm{m} 2 /$ 月; B5为小区绿化率; $\mathrm{B} 6$ 为车位; $\mathrm{B} 7$ 为朝向; $\mathrm{B} 8$ 为装修程度; B9为卧室个数; B10为卫生间个数; B11为焚烧厂到附近小 区的距离, 以 $\mathrm{km}$ 为单位。X1至X11为必要的系数。因本文 的 11 个变量均为有效变量, 所以在软件中选择强行进入法 作回归分析。
表2 特征变量的描述统计。

\begin{tabular}{llllll}
\hline 特征变量 & 样本量 & 极小值 & 极大值 & 平均值 & 标准差 \\
\hline 房龄 & 442 & 3 & 20 & 11.42 & 4.61 \\
装修程度 & 442 & 0 & 3 & 2.23 & 1.16 \\
朝向 & 442 & 0 & 2 & 1.26 & 0.51 \\
车位 & 442 & 0 & 1 & 0.21 & 0.41 \\
绿化率 & 442 & 0.1 & 0.62 & 0.37 & 0.09 \\
物业管理 & 442 & 0.45 & 2.6 & 1.15 & 0.56 \\
卫生间个数 & 442 & 0 & 3 & 1.11 & 0.36 \\
卧室个数 & 442 & 1 & 6 & 2.00 & 0.76 \\
交通条件 & 442 & 2 & 3 & 2.53 & 0.50 \\
生活配套 & 442 & 2 & 3 & 2.83 & 0.38 \\
\hline
\end{tabular}




\begin{tabular}{llllll}
\hline 特征变量 & 样本量 & 极小值 & 极大值 & 平均值 & 标准差 \\
\hline 到焚烧厂距离 & 442 & 411 & 4000 & 1828.2 & 1041.26 \\
修正后的房价 & 442 & 9.25 & 11.26 & 10.63 & 0.34 \\
\hline
\end{tabular}

（2）模型检验将 11 个特征变量在软件中进行分析后 可知, 线性模型中的偏回归系数 $\mathrm{R}=0.661$, 估计的标准误 为 $0.25931, R 2=0.437$, 经过调整后的 $R 2=0.405$, 也就是 说该线性模型可以解释因变量之间的差异百分比达到了 $40.5 \%$, 表明自变量与因变量之间的线性关系比较强, 拟 合效果良好, 这些特征变量可以作为解释房价变化的因素。 根据线性模型的方差分析结果（表4）, 回归方程通过了 显著性检验, 因回归方程的显著性检验值为 0.000 , 小于 0.001 , 表明该回归方程的显著性良好, 所以特征变量与 房价之间的线性关系能够成立。

b. 数据分析

由结论可得模型检验将 11 个特征变量在软件中进行 分析后可知, 线性模型中的偏回归系数 $\mathrm{R}=0.661$, 估计的 标准误为 $0.25931, \mathrm{R} 2=0.437$, 经过调整后的 $R 2=0.405$, 也就是说该线性模型可以解释因变量之间的差异百分比
达到了 $40.5 \%$, 表明自变量与因变量之间的线性关系比较 强, 拟合效果良好。根据线性模型的方差分析结果(表2), 回归方程通过了显著性检验, 因回归方程的显著性检验值 为 0.000 , 小于 0.001 , 表明该回归方程的显著性良好, 所 以特征变量与房价之间的线性关系能够成立。

表3 线性模型的方差分析。

\begin{tabular}{llllll}
\hline 模型 & & 平方和 & 自由度 & F统计量 & 失效概率 \\
\hline \multirow{2}{*}{ 线性 } & 回归 & 10.211 & 11 & & \\
模型 & 残差 & 13.179 & 196 & 13.806 & 0.000 \\
& 总计 & 23.39 & 207 & & \\
\hline
\end{tabular}

c. 回归结果分析

通过回归分析后得到的该线性模型的回归系数见表 5 , 故该特征价格定价模型的回归方程表示为:

$\ln \mathrm{P}=11.438+0.002 \times \mathrm{B} 1-0.033 \times \mathrm{B} 2-0.1 \times \mathrm{B} 3+0.082 \times$ $\mathrm{B} 4+1.212 \times \mathrm{B} 5+0.224 \times \mathrm{B} 6-0.304 \times \mathrm{B} 7+0.017 \times \mathrm{B} 8-0.17 \times$ B9- $0.264 \times \mathrm{B} 10+0.00006377 \times \mathrm{B} 11$

表4 线性模型的回归系数。

\begin{tabular}{|c|c|c|c|c|c|}
\hline \multirow{2}{*}{ 模型变量 } & \multicolumn{2}{|c|}{ 非标准化系数 } & \multirow{2}{*}{$\begin{array}{l}\text { 经过标准化的系 } \\
\text { 数 }\end{array}$} & \multirow{2}{*}{$\mathrm{T}$ 检验 } & \multirow{2}{*}{ 显著性 } \\
\hline & $\mathrm{B}$ & 标准误差 & & & \\
\hline （常量） & 11.438 & 0.236 & & 48.44 & 0.000 \\
\hline 房龄 & 0.002 & 0.006 & 0.029 & 0.329 & 0.743 \\
\hline 装修程度 & -0.033 & 0.017 & -0.114 & -1.944 & 0.053 \\
\hline 朝向 & -0.1 & 0.036 & -0.153 & -2.75 & 0.007 \\
\hline 车位 & 0.082 & 0.055 & 0.100 & 1. 497 & 0.136 \\
\hline 绿化率 & 1. 212 & 0.238 & 0.336 & 5. 097 & 0.000 \\
\hline 物业管理 & 0.224 & 0.051 & 0.375 & 4. 401 & 0.000 \\
\hline 卫生间个数 & -0.304 & 0.073 & -0.324 & -4.179 & 0.000 \\
\hline 卧室个数 & 0,017 & 0.031 & 0.038 & 0.528 & 0.598 \\
\hline 交通条件 & -0.17 & 0.04 & -0.253 & -4.257 & 0.000 \\
\hline 生活配套 & -0.264 & 0.066 & -0.294 & -4.008 & 0.000 \\
\hline 到焚烧厂距离 & 6. $37 \mathrm{E}-005$ & 0.000 & 0.198 & 2. 718 & 0.007 \\
\hline
\end{tabular}

从表 4 可知, 大部分的变量, 如生活配套、交通条件、 小区绿化率、装修程度等对房价都具有正的影响。[3]住 宅小区到最近的焚烧厂的距离对房价的影响是比较符合 假设的, 可以看出, 离焚烧厂越近, 房价受到的影响就越 大。

\section{4. 江桥焚烧厂空间效应实证分析}

通过江桥焚烧厂附近24个住宅小区的房价样本, 以中 房上海住宅指数对住宅价格进行修正, 将其统一归化到 2016年12月。如下图。将样本数据导入软件, 对住宅价格 与距离之间的关系进行定量分析和曲线拟合 (设定住宅价
格为Y, 住宅小区到焚烧厂的距离为X），结果如表5。由 此可知, 住宅价格与住宅小区到焚烧厂的距离之间的二次 模型为:

$$
\mathrm{Y}=31379.124+13.75 \mathrm{X}-0.003 \mathrm{X}^{2}
$$

二次曲线模型的F统计量 5.953 , 大于线性模型的F统 计量 0.021 , 且显著性 $0.003<0.884$, 说明住宅价格和空间 距离之间呈比较明显的二次关系, 而且存在极值点, 其空 间距离最大值为 $2291 \mathrm{~m}$, 这里不收焚烧厂影响的房价为 47134 元 $/ \mathrm{m}^{2}$, 同时即为上海江桥焚烧厂对附近小区的最大 范围。

表5 模型回归系数估计。

\begin{tabular}{|c|c|c|c|c|c|c|c|}
\hline \multirow{2}{*}{ 地区 } & \multirow{2}{*}{ 方程 } & \multicolumn{3}{|c|}{ 模型汇总 } & \multicolumn{3}{|c|}{ 回归系数估计值 } \\
\hline & & $\mathrm{R} 2$ & $\mathrm{~F}$ & 显著性 & 常数 & B1 & B2 \\
\hline 江桥焚烧厂 & 线性二次 & 0.055 & 5.95 & 0.003 & 137.75 & 13.75 & -0.003 \\
\hline
\end{tabular}

最后, 根据已有的条件来量化焚烧厂对附近居民经济 上的损失 $500 \mathrm{~m}$ 处受到焚烧厂的影响的房价为 37504 元 $/ \mathrm{m}^{2}$, 和不收焚烧厂影响的房价相差了 9630 元 $/ \mathrm{m}^{2}$, 而这里有 828 户，每户约有房子 $80 \mathrm{~m}^{2}$ ，最后对 $500 \mathrm{~m}$ 处的居民的影响为
637.89 万元。同理, 对 $800 \mathrm{~m}$ 处的影响为 832.68 万元, 对 $1000 \mathrm{~m}$ 处的影响为 172.93 万元, 对 $1200 \mathrm{~m}$ 处的影响为 307.58 万元。再将其加总除以 70 年, 得到每年的损失为 27.87 万 元。 [4] 


\section{3. 附近居民的健康损益函数}

\section{1. 规模为 $1500 \mathrm{t} / \mathrm{d}$ 的垃圾焚烧厂对附近居民健康影响}

表6为非正常工况下 $1500 \mathrm{t} / \mathrm{d}$ 垃圾焚烧厂周围居民的 致癌风险。 5 ] 由表可知, 最大落地浓度点处的总致癌风 险值为 $1.70 \times 10^{-5}$, 存在致癌风险但属于可接受的范围, 主要风险物质为 $\mathrm{Cd}$ 。

非正常工况下 $1500 \mathrm{t} / \mathrm{d}$ 垃圾焚烧厂周围居民的非致 癌风险如表3-18所示。最大落地浓度点成人的总非致癌风 险值为 $9.70 \times 10^{-2}$, 儿童的非致癌风险值为 $1.79 \times 10^{-1}$, 不 存在非致癌风险。[6]比较各种污染物对总非致癌风险的 贡献发现, 非致癌风险 $\mathrm{Hg}>\mathrm{Pb}>$ 二噁英 $>\mathrm{Cd}$ 。[7]

表6 $1500 \mathrm{t} / \mathrm{d}$ 垃圾焚烧厂周围居民的致癌风险。

\begin{tabular}{llll}
\hline \multirow{2}{*}{ 位置 } & \multicolumn{2}{c}{ 致癌风险 } & \multirow{2}{*}{ 总致癌风险 } \\
\cline { 2 - 3 } & 二噁英 & $\mathrm{Cd}$ & $1.70 \times 10^{-5}$ \\
\hline $\begin{array}{l}\text { 最大落地点 } \\
300\end{array}$ & $3.94 \times 10^{-6}$ & & $1.70 \times 10^{-5}$ \\
500 & & $7.23 \times 10^{-6}$ & $7.23 \times 10^{-6}$ \\
800 & $1.68 \times 10^{-6}$ & $2.72 \times 10^{-6}$ & $2.72 \times 10^{-6}$ \\
1000 & $6.34 \times 10^{-7}$ & $1.56 \times 10^{-5}$ & $1.56 \times 10^{-5}$ \\
1200 & $3.62 \times 10^{-6}$ & $6.40 \times 10^{-6}$ & $6.55 \times 10^{-6}$ \\
\hline
\end{tabular}

\section{2. 焚烧厂对附近居民的损益}

(1) 对于环境污染损害赔偿的赔偿范围的建议 对当事人提出的损害赔偿, 笔者认为可以从以下几方 面予以考虑:

人身损害赔偿。由于环境污染造成人身伤害的, 赔偿 的范围包括 $[8]$ :

a. 医疗费 (包括继续治疗费)；

b. 误工费;

c. 护理费;

d. 交通费;

e. 住宿费;

f. 住院伙食补助费;

g. 营养费;

h. 残疾赔偿金;

i. 被扶养人生活费;

j. 残疾辅助器具费;

$\mathrm{k}$. 死亡赔偿金和丧葬费。

财产损害赔偿, 环境污染造成的损害赔偿包括三方面: 一、直接经济损失, 是指由于环境污染直接造成设施的破 坏、产量或质量下降所引起的损失, 该损失一般是可以用 市场价格来计算的。二、间接经济损失，是指环境污染侵 害受害人所有的财物, 致使受害人在一定范围内的未来财 产利益的损失。三、恢复到损害前状态所需要的费用。

精神损害赔偿, 由于环境污染造成人身伤害的, 受害 人或死者的其近亲属依照《最高人民法院关于确定民事侵 权精神损害赔偿责任若干问题的解释》第一条的规定, 可 以提出精神损害赔偿。[1]

(2) 对于赔偿标准的建议

对于人身损害的赔偿标准，认为可以参照《最高人民 法院关于审理人身损害赔偿案件适用法律若干问题的解
释》的规定予以确定;对于污染环境造成受害人健康的潜 在危害，如人体功能减退、早衰等，虽未经医治，尚没有 支出医疗费等, 也应当予以适当赔偿, 以补偿人体的潜在 损害。[1]

环境污染是生活中常有的一些情形, 然而在受到污染 之后造成的损害却难以拿到赔偿, 在生活中都是因为, 环 境污染是很难举证的, 也就是说举证是有一定困难的, 并 且在涉及赔偿这方面, 法律对企业环境污染赔偿标准的具 体金额以及都是没有具体的规定的, 需要根据实际情况进 行分析然后进行赔偿。

(3) 根据已知的条件量化健康损失

结合距离焚烧厂的距离不同所存在的居民人口数, 以 及总致癌风险来计算出最终居民一方每年可能致病的人 数。在 $500 \mathrm{~m}$ 的范围有 828 户, $500 \mathrm{~m}$ 到 $800 \mathrm{~m}$ 的范围有 5760 户， $800 \mathrm{~m}$ 到 $1000 \mathrm{~m}$ 的范围中有 4320 , 在 $1000 \mathrm{~m}$ 到 $1200 \mathrm{~m}$ 的范围中 有 2880 户。每户约有三口人, 则可以计算出其每年可能治 病的人数为 0.33 个人。根据现在的早期发现癌后治疗费用 至少为 12 万元, 那么每年对附近居民可能造成的健康上的 损失约为 3.96 万元。

\section{4. 附近居民的心理的损益}

马斯洛需求层次理论是人本主义科学的理论之一, 由 美国心理学家亚伯拉罕·马斯洛在 1943 年在《人类激励理 论》论文中所提出。[9]书中将人类需求像阶梯一样从低 到高按层次分为五种, 分别是: 生理需求、安全需求、社 交需求、尊重需求和自我实现需求。在上海地区的人民生 活水平普遍较高, 所以都是追求安全需求以上的需求。 [10]

虽然心理上的损失无法量化出来，但是人可以对不同 的情况不同的心里感受进行排序。例如情况一是在未被告 知的情况下, 被其他人侵犯了自己正常的安全需求。情况 二是在通过交流的情况下, 自己愿意妥协让步的受到一些 侵犯自己的安全需求。这二种情况具体能够相差多少无法 量化, 但是可以明确的知道第二种情况人的心理损失要小 于第一种情况。

\section{5. 解决方案}

（1）要想将垃圾焚烧的 “邻避效应” 的问题给解决 掉就必须认识到不能一味的身避问题, 担心居民会抗议。 而是要焚烧厂先要有坚定的信念去解决问题。

（2）在解决问题的同时不能单纯的依靠蛮力去做, 同时要考虑到居民的经济上和心理上的损失。不但要对受 到经济损失的居民进行补贴, 还要顾及到他们心理上的感 受。让附近的居民参与到焚烧厂的建设中来, 一方面是尊 重了居民的知情权, 另外一方面是让居民了解到焚烧厂并 没有想象中那样减轻心理抗拒。

（3）如果想要在较短时间内解决城市的垃圾焚烧问 题, 首先应该选址在较为偏远的地区。其次, 不能轻易的 妥协放弃建造焚烧厂。而是要先强硬的将焚烧站建立起来 
然后再对焚烧厂的污染物进行治理, 同时对附近居民进行 安抚。

（4）焚烧厂应该更多让附近的居民有知情权，时时 的对外公布焚烧厂的废气排放情况。同时也让附近的居民 参与到焚烧厂的建造和监督中来。从而重新赢得居民的信 任，使得居民对焚烧厂的心理上的影响降到最低。

（5）如果不是希望在很快的速度将焚烧厂建立起来而 是更多的考虑人文关怀的话, 那么最优的办法的在选址的 时候就开始和附近的居民讨论。怎么建造、怎么监督等一 系列的问题都是双方讨论的重点。这样虽然时间跨度比较 长但是不是让双方造成直接的冲突, 一举多得。

\section{6. 结论}

结合特征价格去量化焚烧厂对其附近居民的影响，以 及参考了现有的医疗条件和医疗费用情况来评估出焚烧 厂对附近居民的健康上的影响。[11] 虽然没法正面的量化 出焚烧厂对居民心理上的影响, 但是人可以对不同的情况 不同的心里感受进行排序。能全面地分析出在焚烧厂附近 的居民受到的来自经济、健康、心理上的影响的大小。

从这些量化的结果可以看出, 如果政府想要建立一个 焚烧厂时, 让附近的居民受到了多大的损失。从而来在居 民起来抗议的时候能够比较准确的了解这种损失来对他 们进行一系列的补贴。反之, 居民也可以很清晰的看出自 己所受到的危害有多大, 以此为基础向政府发出请求其改 善焚烧厂周围居民生活环境。

\section{参考文献}

[1] 吴军. 我国人身伤害赔偿的司法鉴定现状与思考 [J]. 中国 司法鉴定，2002（2）:45-47。

[2] 石忆邵, 付伟.上海自贸区对周边住房价格的空间影响效应 分析 [J]. 上海国土资源，2016, 37 (1):33-38。

[3] 叶发强. 生活垃圾焚烧机理分析及试验研究 [D]. 哈尔滨工 业大学, 2009。

[4] 郭万翠. 沽源县生态系统服务价值变化及土地利用优化研 究 $[J]$. 自然地理学, 2008。

[5] 徐海云. 全球生活垃圾焚烧处理发展分析 [J]. 城市管理与 科技, 2014(5):21-24。

[6] 林佳佳, 王维, 居婕, 刘晓否, 孙华. 无锡市锡山区棕 (褐) 地环境风险评价研究》 [J]. 中国环境科学, 2013 (4) : 748-753。

[7] 邹滨, 曾永年, Bell jaminF. Zhan杨令宾, 张鸿辉. 城市水 环境健康风险评价》 [J]. 地理与地理信息科学, 2009 (2) : $94-98$ 。

[8］王华杰. 试论环境污染侵权的民事赔偿 $[J]$. 河南商业高等 专科学校学报, 2008 (3) : 78-82。

[9] 张海南, 李琴. 马斯洛需求理论视角下权力腐败的原因及对 策探讨 $[J]$. 人间, 2015（35）:79-79。

[10] 陆慧. 经济发达地区高等学校教师的需求研究 [J]. 大学教 育科学, 2010 (3) :61-66。

[11] 朱天骏. 垃圾焚烧厂对周边住宅价格影响的特征价格研究 [D]. 合肥工业大学, 2015。 